\title{
The Search for Accuracy in Neonatal Volume-Targeted Ventilation: For Whom, the Manufacturer, the Physician, or the Patient?
}

Since the development of volume-targeted ventilation, which has become the most recommended mode for lungprotective ventilation in neonates, many investigators (sometimes driven by corporate interests) have measured the accuracy of tidal volume $\left(\mathrm{V}_{\mathrm{T}}\right)$ delivery for various ventilators primarily by using bench-testing while simulating various respiratory system conditions, including the introduction of an airway leak (ie, endotracheal tube [ETT] leakage). Such studies have repeatedly shown some discrepancies between various ventilators for desired and effectively measured $\mathrm{V}_{\mathrm{T}}$, observations that have often been used as an argument for some inconsistent outcomes of clinical studies that investigate the superiority of volume-targeted ventilation versus various modes of pressure-controlled ventilation.

What might be the real clinical advantage of volumetargeted ventilation? It is true that volume-targeted ventilation has shown some superiority over pressure-control modes in meta-analytic outcome analysis for death and/or chronic lung disease, although the quality of data varied from moderate to low and there were important heterogeneity among individual studies (of note, all of them were unblinded). ${ }^{1}$ One more advantage of volume targeting would be to have stable minute ventilation and, therefore, stable $\mathrm{P}_{\mathrm{aCO}}$ values, which allow for a better neurodevelopmental outcome. In fact, $\mathrm{CO}_{2}$ stability and a lower incidence of hypocarbic events have been shown to be more achievable with volume-targeted ventilation than with synchronized intermittent pressure ventilation. However, this was only the case for infants at $>25$ weeks of gestation. ${ }^{2}$

Targeted $\mathrm{V}_{\mathrm{T}}$ values are commonly in the range of 4 to $6 \mathrm{~mL} /$ $\mathrm{kg}$, with the recommendation to use even larger $\mathrm{V}_{\mathrm{T}}$ values in very small premature infants because of a proportionally larger instrumental dead space from the ETT and pneumotachograph inserted at the Y-piece. ${ }^{3}$ However, there exists no single study that investigated in the best protective VT (in $\mathrm{ml} / \mathrm{kg}$

The author discloses relationships with SLE Ltd, Maquet, Stephan GmbH, imtmedical, and Sentec.

Correspondence: Peter C Rimensberger MD, Service of Neonatology and Pediatric Intensive Care, Hôpital des Enfants, HUG, 6, Rue Willy-Donze, Geneva, CH-1211, Switzerland. E-mail: peter.rimensberger@hcuge.ch.

DOI: $10.4187 /$ respcare.06962 bodyweight) range in neonates and/or infants who presented with respiratory distress. Therefore, and with the concept of a "safe" range of $\mathrm{V}_{\mathrm{T}}$, the question becomes, to what extent accuracy of $\mathrm{V}_{\mathrm{T}}$ measures for volume-targeted ventilation as the primary control parameter, regulated either on the expired, the inspired, or a combination of both, is really of clinical relevance. Note that it has also been shown that the displayed, and for volume-targeted ventilation algorithms used, $\mathrm{V}_{\mathrm{T}}$ from the ventilator may differ up to $10 \%$ from measures by a device independent pnumotachograph, ${ }^{4}$ an observation that, in general, questions the accuracy of measures of very small $\mathrm{V}_{\mathrm{T}}$.

\section{See the Original Study on Page 361}

The question to ask in the end, is not which ventilator is the best machine to regulate the $\mathrm{V}_{\mathrm{T}}$ most precisely under any respiratory system condition, but what would be best in terms of respiratory support for the patient's clinical condition. In that, the study by DiBlasi et $\mathrm{al}^{5}$ in this issue of Respiratory CARE looked closer to the patient while investigating in an in vivo study (sedated but spontaneous breathing animals) the physiologic effects of different neonatal volume-targeted algorithms and $\mathrm{V}_{\mathrm{T}}$-measurement concepts of 3 neonatal ventilators. They addressed not only $V_{T}$ measures and the accuracy of volume-regulation algorithms but also ventilation efficiency, as measured by gas exchange, trigger efficiency, and imposed work of breathing in the presence or absence of a graduated ETT leak.

One machine (Babylog VN500, Draeger, Lubeck, Germany) that offers adaptive leak compensation by using complex and proprietary algorithms that take into account the inspired and expired $\mathrm{V}_{\mathrm{T}}$, managed to keep the exhaled $\mathrm{V}_{\mathrm{T}}$ value, as measured by an independent flow meter, within a 5\% error of the preset value for both leak and no leak conditions. This resulted in stable $\mathrm{P}_{\mathrm{aCO}}$ values, despite the observation that the spontaneous breathing rate decreased by $12 \%$, which resulted in a reduction of calculated minute ventilation by $8 \%$. This might suggest, as the authors hypothesized, that, in the presence of leak when the machine adds additional flow, $\mathrm{CO}_{2}$ might be better washed out from the airways, similar to the concept of tracheal insufflation or high-flow nasal cannula therapy. 


\section{EDITORIALS}

Whether this effect might increase the risk of hypocarbia in a subject without spontaneous breathing efforts remains unclear. With the second device (AVEA, CareFusion, Yorba Linda, CA), when regulating on the exhaled $\mathrm{V}_{\mathrm{T}}$ only, the $\mathrm{P}_{\mathrm{aCO}}$ values significantly decreased in the presence of an ETT leak, which suggests some leak overcompensation and the effect of $\mathrm{CO}_{2}$ washout. Interestingly, with the third device (Servo-i, Maquet/Getinge, Solna, Sweden), which offers, in the model and version tested, no leak compensation and regulates on inspired $V_{T}$, which will lead in the presence of an ETT leak to a reduction of the $\Delta$-pressure (ie, pressure above PEEP) to keep the inspired $V_{T}$ constant. In this scenario, the measured exhaled $\mathrm{V}_{\mathrm{T}}$ decreased below the target as would be expected, while spontaneous respiratory rates slightly increased, which resulted in unchanged $\mathrm{P}_{\mathrm{aCO}}$ values.

Yet, it seems that two of the tested devices, despite complete opposite regulation algorithms, allowed the spontaneous breathing animals to maintain $\mathrm{P}_{\mathrm{aCO}}$ stability in the presence or absence of an ETT leak. But what is the price that the subjects had to pay for this consistency? Whereas work of breathing as measured by the pressure-rate product calculated from esophageal pressure measures was equal for all 3 devices for the no-leak condition, it decreased with the Babylog VN500 and the AVEA when a leak was introduced. This would suggest that, in patients in whom spontaneous breathing efforts are supported, it would be best to search for an important ETT leak when using a device that regulates $\mathrm{V}_{\mathrm{T}}$ leak compensated on the exhaled or on both inspired and exhaled $V_{\mathrm{T}}$. Whereas, with a device that regulates the inspired $V_{T}$ without leak compensation, the leak condition would not matter.

However, such interpretation of these findings might be flawed because, besides the various concepts and algorithms used for targeting a $\mathrm{V}_{\mathrm{T}}$ for these 2 devices, asynchrony events (ie, inspiratory trigger failure), as observed with and without a leak condition with the Servo-i, might have had a major effect on imposed work of breathing. This observed important patient-ventilator asynchrony might be attributed to the fact that the flow sensor used for regulating $\mathrm{V}_{\mathrm{T}}$ adjustments in this device is positioned within the device. Note, the manufacturer of the Servo-i, which does not have a leak compensation algorithm, does explicitly recommend not to use the volume-target mode in the presence of a major leak (ie, $\geq 50 \%$ ). Therefore, it would have been desirable to compare ventilators of the same generation, where, at least in areas that accept CE-market (European Union approval), technology has for some years already been available with the Servo-u or Servo-n (Maquet/Getinge), which both offer leak compensation and regulate $V_{T}$ on measures from a pneumotachograph at the Y-piece. Also, the latter may probably allow a reduction in asynchrony events. But this needs to be investigated.

All these observations and thoughts illustrate not only the complexity of comparative measures among devices but also that the individual's physiologic response to pos- itive-pressure ventilation is more complex than we tend to think when testing devices on the bench. However, in the end, the essential questions that the clinician has to ask are the following:

1. What type and amount of support might be the best for my patient's actual disease condition?

2. How can my patient adapt to and interact optimally with the ventilator?

3. To what extent does the accuracy of numbers and measures matter for the patient?

In trying to partially respond to these questions, we need to better define what the ultimate outcome goals will be and what price to pay will be acceptable for a patient. In this search, the first maxim should be to cause no further harm, the second to improve short- and long-term outcomes, and the third to ensure patient comfort. This no harm and improve outcome concept that focused for many years on airway pressure limitation to protect the lung moved toward targeting $\mathrm{V}_{\mathrm{T}}$ to protect the lung and ensure better $\mathrm{P}_{\mathrm{aCO}}$ stability while trying to minimize alterations in cerebral perfusion. Targeting a specific $\mathrm{V}_{\mathrm{T}}$ calls for high accuracy in $\mathrm{V}_{\mathrm{T}}$ measures and assumes not only that $\mathrm{V}_{\mathrm{T}}$ variability would be bad but that it also assumes that we know the specific best $\mathrm{V}_{\mathrm{T}}$ for the patient's actual respiratory system conditions. However, for the latter, we do not have any data that would guide us when ventilating and/or assisting breathing in a neonate. Furthermore, irregular breathing with variable spontaneous $\mathrm{V}_{\mathrm{T}}$ and variation of breathing frequency is highly physiologic in the preterm infant. Therefore, one might raise a further question, namely, whether some variability in $\mathrm{V}_{\mathrm{T}}$ during ventilatory support would be desirable or beneficial for the patient. ${ }^{6,7}$

Patient-ventilator asynchrony is a second topic that has become a modern topic for device testing and altered since the appearance of neural adjust ventilatory assist. Although trigger and cycling asynchronies might increase the work of breathing of the patient and, in some conditions, call for more sedation, it has not conclusively been shown yet that minimizing asynchrony events would lead to better patient outcomes. However, better synchrony seems to be associated with better patient comfort.

Increased work of breathing and poor gas exchange are certainly 2 factors that might contribute to the severity of respiratory failure and an ongoing need for mechanical ventilation in premature infants. It would be highly desirable that ventilators are capable of delivering appropriate $\mathrm{V}_{\mathrm{T}}$ and that they allow for effective triggering and cycling. However, this study by DiBlasi et $\mathrm{al}^{5}$ did not help to confirm that the call for high accuracy and precision of $\mathrm{V}_{\mathrm{T}}$ targeting, with almost a zero error allowance in measures and delivery of $\mathrm{V}_{\mathrm{T}}$, would be so essential for the patient and his or her outcome. It could be argued for this study 


\section{EDITORIALS}

that the essential parameter that could explain the observed differences among ventilators would only be the observed patient-ventilator asynchrony. Therefore, we do not know yet whether it really matters for the patient if inspiratory, expiratory, or adaptive $\mathrm{V}_{\mathrm{T}}$ targeting algorithms are used. Also we do not know yet how much precision in $\mathrm{V}_{\mathrm{T}}$ measures and $\mathrm{V}_{\mathrm{T}}$ regulation algorithms will finally be required to have an impact on patient outcome.

Peter C Rimensberger

Service of Neonatology and Pediatric Intensive Care

Department of Pediatrics

University Hospital and University of Geneva

Geneva, Switzerland

\section{REFERENCES}

1. Klingenberg C, Wheeler KI, McCallion N, Morley CJ, Davis PG. Volume-targeted versus pressure-limited ventilation in neonates. Cochrane Database Syst Rev 2017;10:CD003666.
2. Cheema IU, Sinha AK, Kempley ST, Ahluwalia JS. Impact of volume guarantee ventilation on arterial carbon dioxide tension in newborn infants: a randomised controlled trial. Early Hum Dev 2007;83(3):183-189.

3. Sant'Anna GM, Keszler M. Developing a neonatal unit ventilation protocol for the preterm baby. Early Hum Dev 2012;88(12): 925-929.

4. Vignaux L, Piquilloud L, Tourneux P, Jolliet P, Rimensberger PC. Neonatal and adult ICU ventilators to provide ventilation in neonates, infants, and children: a bench model study. Respir Care 2014;59(10): 1463-1475.

5. DiBlasi RM, Kearney CN, Hotz JC, Salyer JW, Poli JA, Crotwell DN and Hartmann SM. Physiologic effects of 3 different neonatal volumetargeted ventilation modes in surfactant-deficient juvenile rabbits. Respir Care 2019;64(4):361-371.

6. Pillow JJ, Musk GC, McLean CM, Polglase GR, Dalton RG, Jobe AH, Suki B. Variable ventilation improves ventilation and lung compliance in preterm lambs. Intensive Care Med 2011;37(8):1352-1359.

7. Bartolák-Suki E, Noble PB, Bou Jawde S, Pillow JJ, Suki B. Optimization of variable ventilation for physiology, immune response and surfactant enhancement in preterm lambs. Front Physiol 2017; $8: 425$. 